\title{
Effect of Medication Education on Drug Adherence with Mental Disorders: A Clinical Trial Study
}

\author{
Efat Sadeghian ${ }^{1}$, Mina Nezafatdoost ${ }^{2}$, Lily Tapak ${ }^{3}$, Farshid Shamsaei ${ }^{4, *}$,
}

\author{
${ }^{1}$ Assistant Professor, Chronic Diseases (Home Care) Research Center, Hamadan University of Medical \\ Sciences, Hamadan, Iran \\ ${ }^{2}$ Msc Student of Psychiatric Nursing, Faculty of Nursing and Midwifery, Hamadan University of Medical \\ Sciences, Hamadan, Iran \\ ${ }^{3}$ Assistant Professor, Department of Biostatistics, School of Public Health Sciences Research Center, Hamadan \\ University of Medical Sciences, Hamadan, Iran \\ ${ }^{4}$ Associate Professor, Mother and Child Care Research Center, Hamadan University of Medical Sciences, \\ Hamadan, Iran \\ * Corresponding author: Farshid Shamsai, Associate Professor, Mother and Child Care Research Center, \\ Hamadan University of Medical Sciences, Hamadan, Iran. E-mail: shamsaei68@yahoo.com
}

Received: 29 Sep 2018

Accepted: 21 Dec 2018

Abstract
Introduction: One of the main goals of treatment in patients with mental disorders is compliance with drug
therapy because non-compliance of the drug causes relapse of the disease and disrupts the treatment process.
The purpose of this study was to investigate the effect of pharmacotherapy training on drug availability in
patients with mental disorders.
Methods: In this clinical trial study, 80 patients with Mental Disorders diagnosed in Farshchian Hospital in
Hamadan, Iran, from October to February 2017 participated. Participants in the study were randomly
divided into two experimental (40) and control (40) groups. In the intervention group, medication
education was administered in four sessions of an hour in group (each group of 10$)$. The control group
received routine nursing care. Patients' adherence with drug therapies was checked by using the checklist for
observing drug compliance before and after the intervention. Data analysis was performed using descriptive
and inferential statistics in SPSS software version 24 .
Results: The two groups did not differ significantly in terms of personal characteristics $(\mathrm{P}>0.05)$. Mean
and standard deviation of drug compliance score before and after intervention in experimental group was
$0.55 \pm 4.03$ and control group was $1.50 \pm 4.34$, which did not show significant difference $(\mathrm{P}=0.306)$. After
the intervention, the difference in mean and standard deviation of drug adherence score was statistically
significant in the experimental group (5.02 \pm 3.27$)$ and control (1.17 \pm 3.53$)(\mathrm{P}<0.001)$.
Conclusions: Medication education interventions have had a positive effect on drug dependence in patients
with psychiatric disorders. Therefore, it is suggested that more attention be paid to educating patients about
nursing care in nursing care programs.
Keywords: Education, Medication Therapy, Mental Patients, Medication Adherence (c) 2018 Iranian Nursing Scientific Association (INA) 


\title{
تأثير آموزش دارو درمانى بر تبعيت دارويى بيماران با اختلالات روانى: يك مطالعه كار آزمايى بالينى
}

\author{
عفت صادقيان '، مينا نظافت دوست ؟، ليلى تاياك ؟ّ، فرشيد شمسايى
}

' 'استاديار، كروه يرستارى، مركز تحقيقات بيمارهاى مزمن (مراقبت در منزل)، دانشكاه علوم يزشكى همدان، ايران

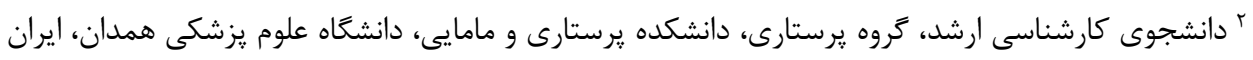

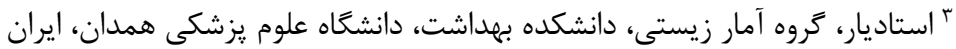

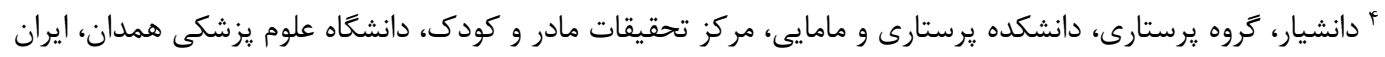

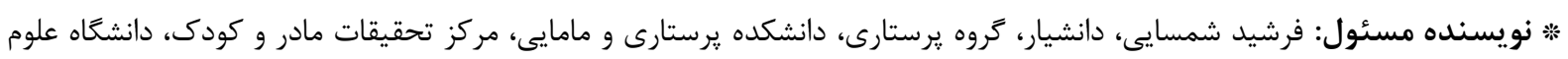
يزشكى همدان، ايران. ايميل: shamsaei68@yahoo.com

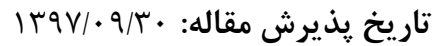

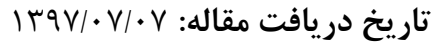

جكيده

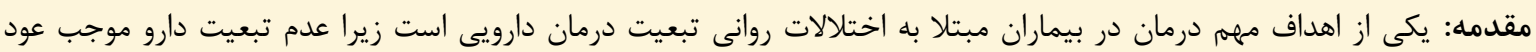

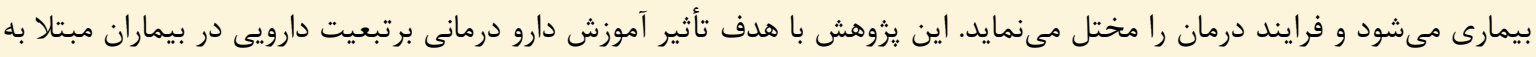
اختلالات روانى بسترى انجام شد.

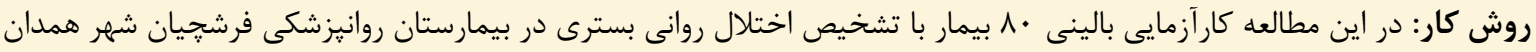

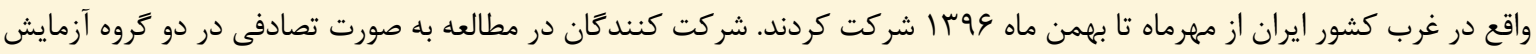

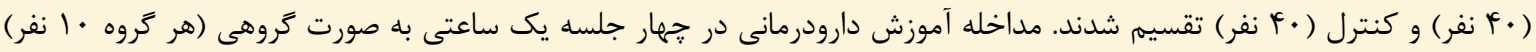

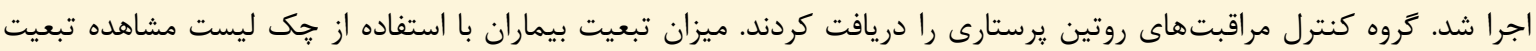

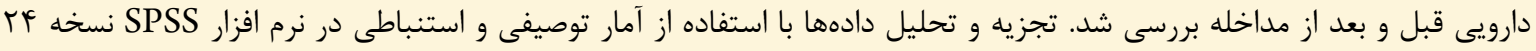
انجام كرفت.

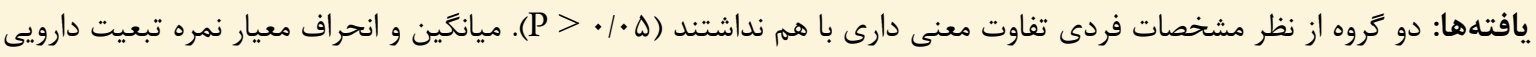

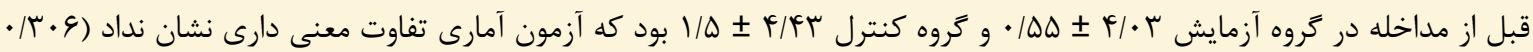
ل

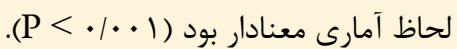

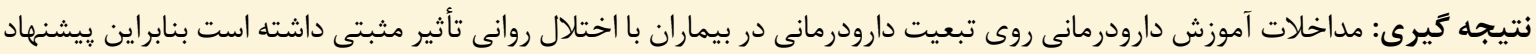

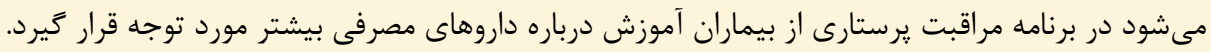

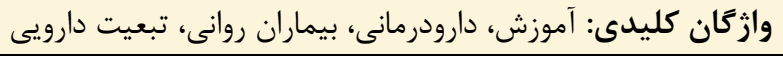

تمامى حقوق نشر براى انجمن علمى برستارى ايران محفوظ استى دانيمان.

مقدمه

اغلب Fام اول در درمان در مرحله حاد بيمارى و همجنين به عنوان

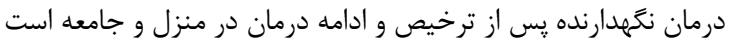

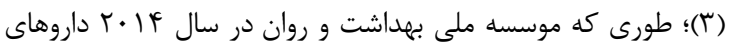

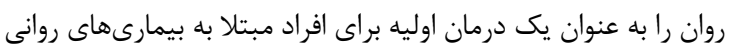

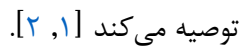

در حال حاضر بيمارىهاى روانى به دو صورت جسمى و روانى درمان

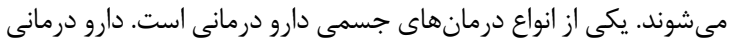

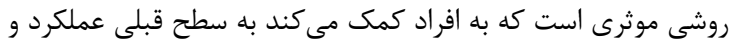

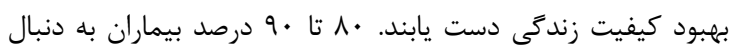

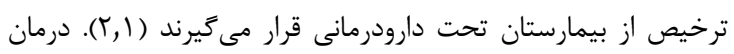

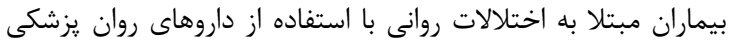


دارو درمانى و تشويق بيماران به تبعيت از درمان دارند. با استفاده از

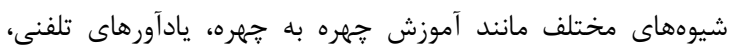

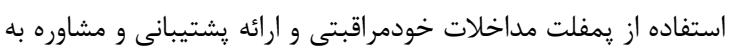

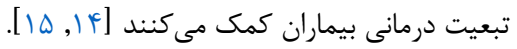

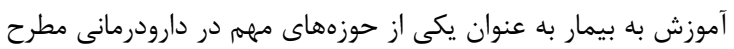

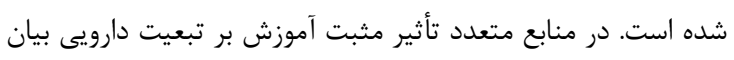

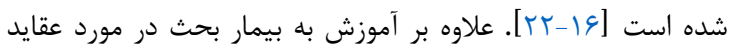

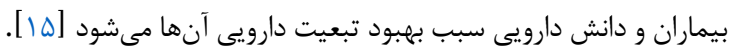

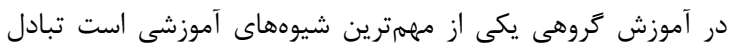

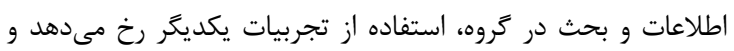

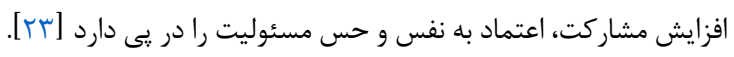

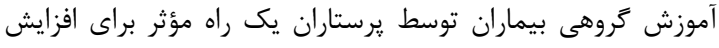

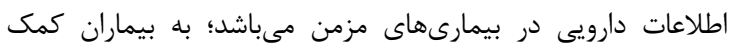

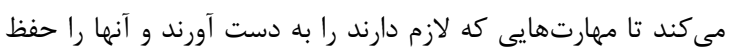

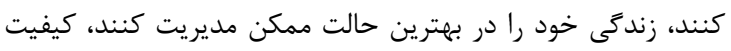

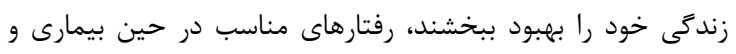

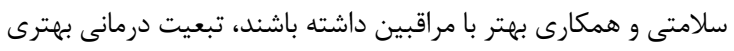

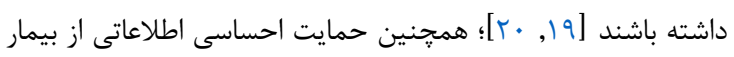

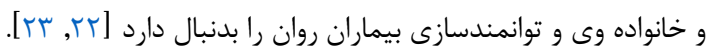

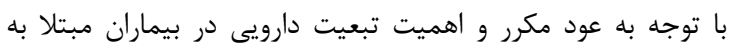

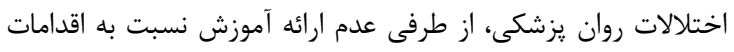

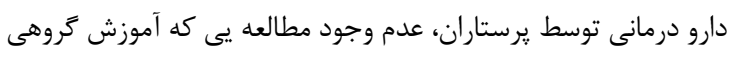

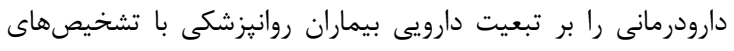

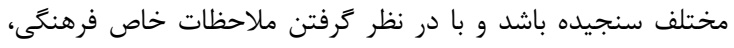
بومى و اقتصادى اين مطالعه با هدف تعيين تأثير آموزش دارئ دارو درمانى فرئي

$$
\text { بر تبعيت دارويى بيماران روان انجام شد. }
$$

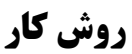

اين يزوهش يك مطالعه كارآزمايى بالينى است و محيط مطالعه بيمارستان روانيزشكى فرشحيان شهر همدان واقع در غرب كشور ائر ايران

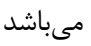

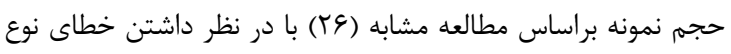

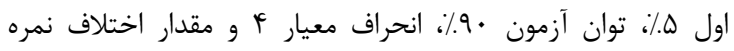

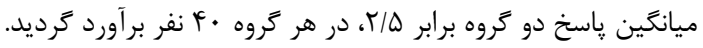

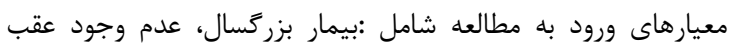
ماندَى ذهنى، تشخيص اختلالات اسكيزوفرنى، اسكيزوافكتيو، اختلال

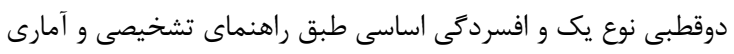

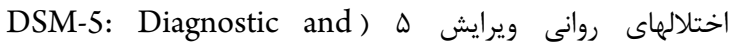
Statistical Manual of Mental Disorders

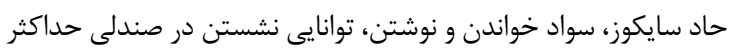

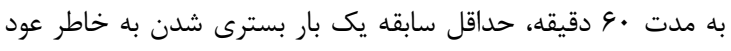

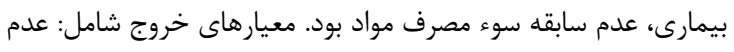

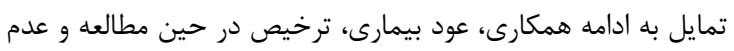

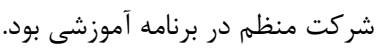

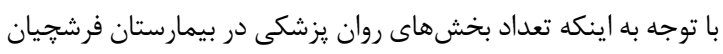

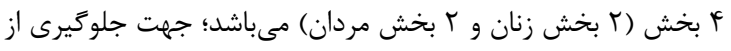

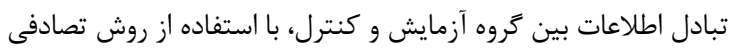

يكى از بزر كترين مشكلات در درمان دارويى بيماران مبتلا به اختلالات

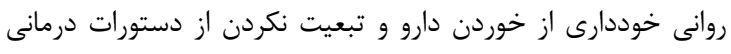

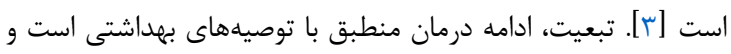

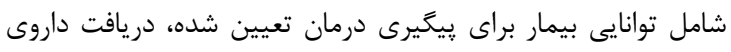

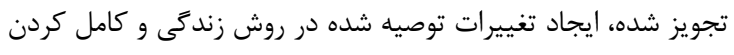

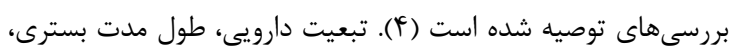

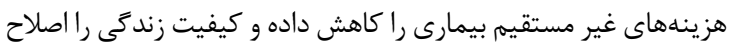

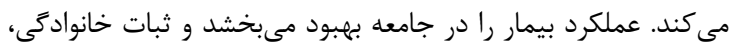

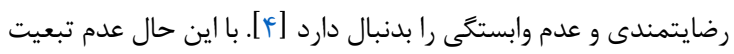

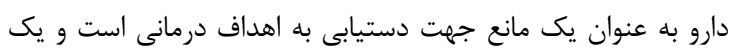

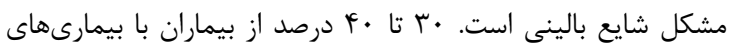
مزمن رزيم دارويى خود رار رعايت نمى كنند [ه]]

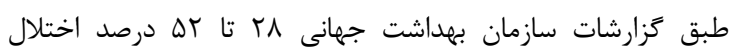

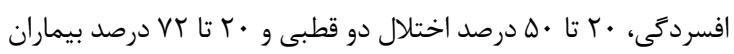

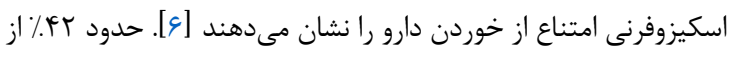

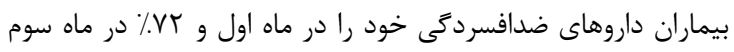

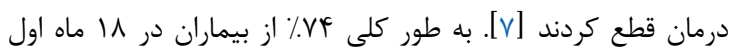

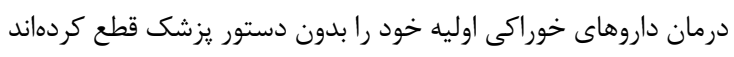

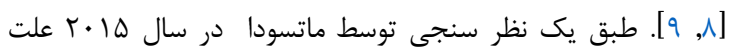

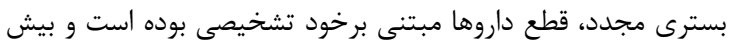

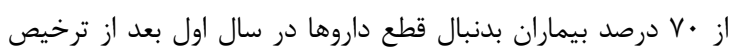

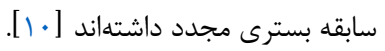

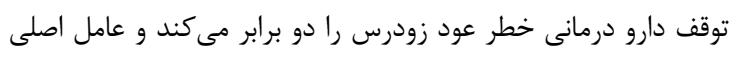

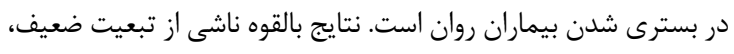

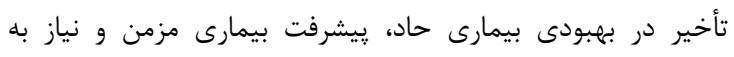

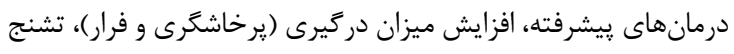

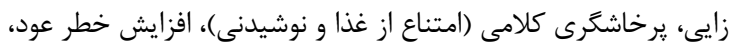

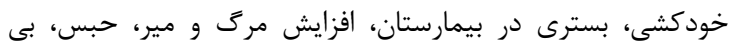

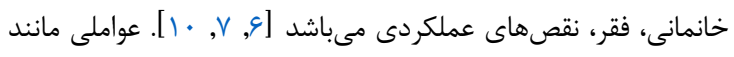

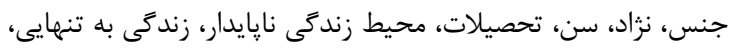

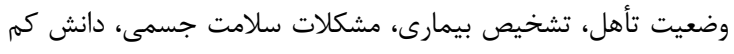

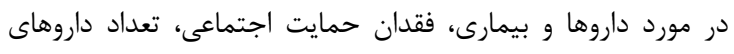

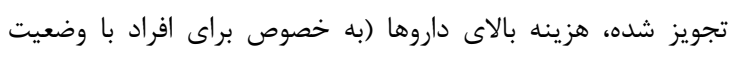

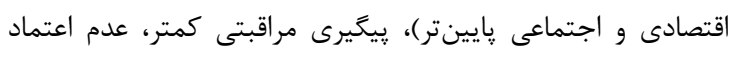

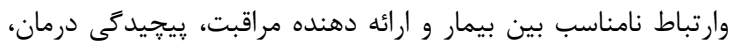

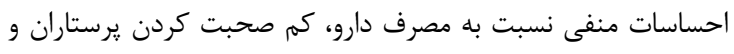

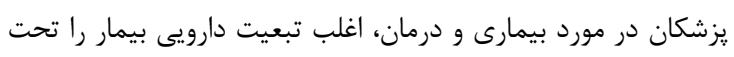

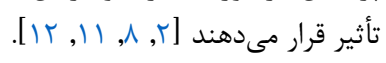

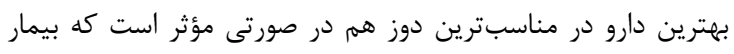

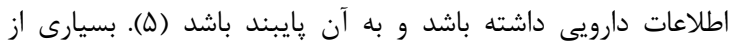

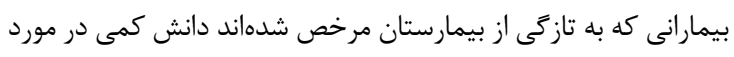

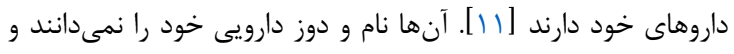

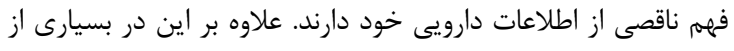

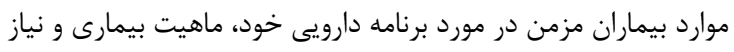

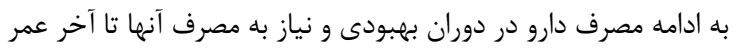

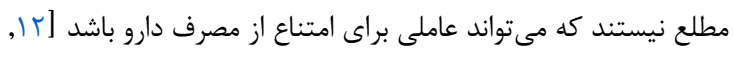

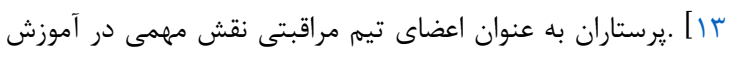




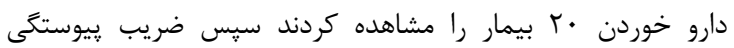

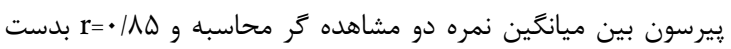
برنامه آموزشى براى هر كروه به شيوه سخنرانى (سمعى و بصرى)،

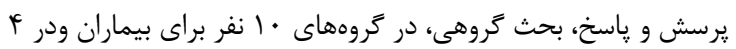

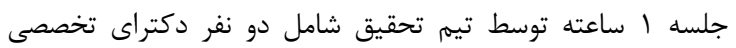

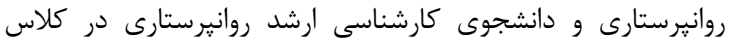

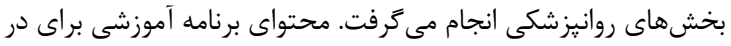

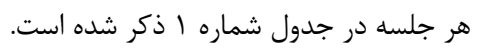

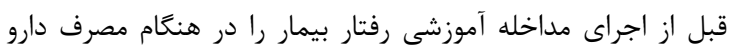

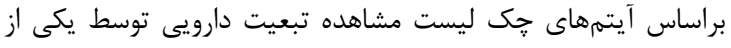

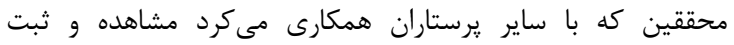

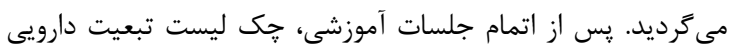

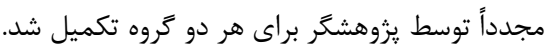

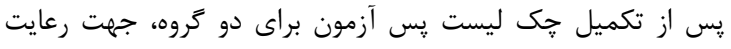

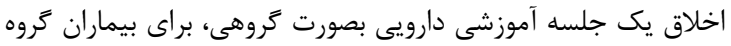
شاهد نيزبر گزار شد.
ساده (يرتاب سكه) دو بخش (يكى از بخش زنان و يكى از بخشهاى

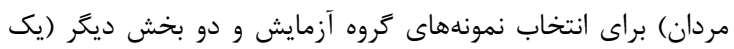

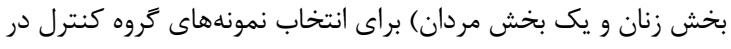

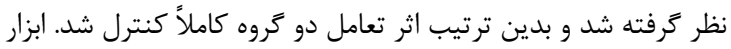

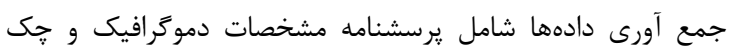
ليست تبعيت دارويى بودند.

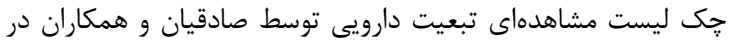

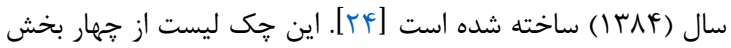

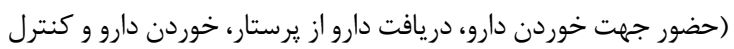

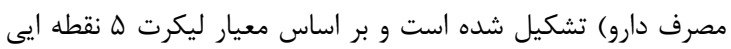

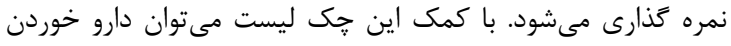

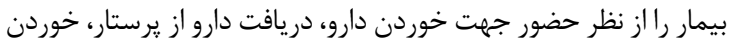

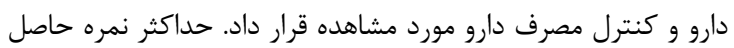

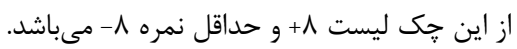

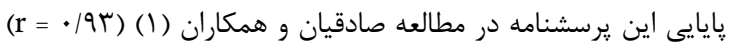

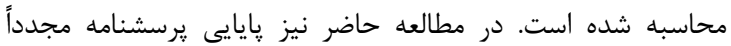

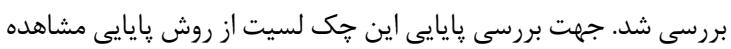

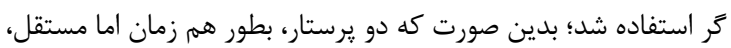

جدول ا: فهرست محتواى جلسات آموزش دارو به بيماران روان

جلسه اول فيرست

معارفه و آشنايى و بيان اهداف آموزشى

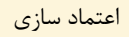

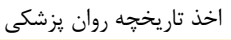
تشويق به بيان تجربيات مصرف دارو

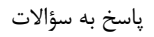

جلسه دوم

آشنايى با بيمارى هاى روان بزشكى

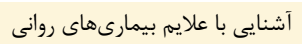

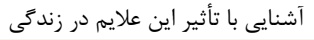

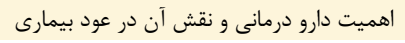

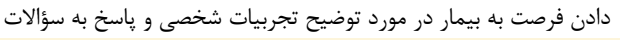

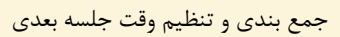

جلسه سوم

يرسش و پاسخ درمورد محتواى جلسه قبلى

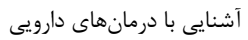
عوارض مصرف داروها فوايد مصرف داروها

آشنايى با دوز مصرفى و مدت مصرف داروها

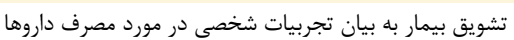

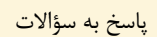
جمع بندى و تنظيم وقت جلسه بعدى بلات جلسه جههارم يرسش و پاسخ از محتواى جلسه قبلى آشئ آشنايى با مراقبتهاى مربوط باز مجتواي داروهل

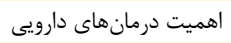

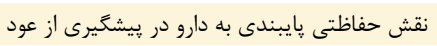

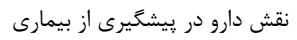
نقد، بررسى و جمع بندى دارو دري بيرى از بيمال يرسش و ياسخ ارزيابى نهايى نيخ باسي 
به ثبت رسيده است و كليه ملاحظات اخلاقى از جمله اخذ مجوز از

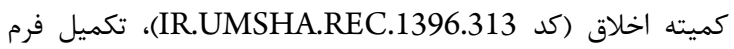

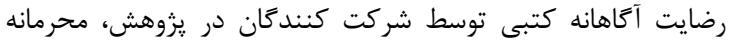

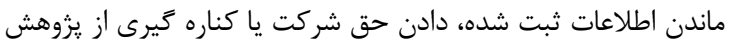
به نمونهها، مورد توجه قرار كرفته است.
جهت تجزيه و تحليل دادهها از نرم افزار آمارى SPSS نسخه

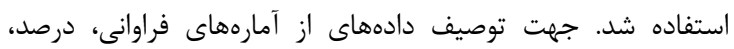

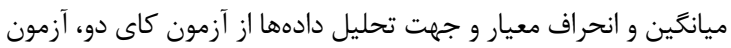

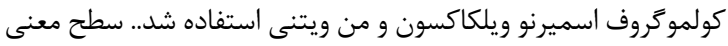

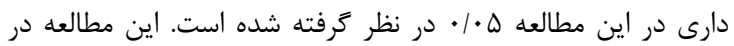

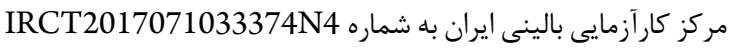

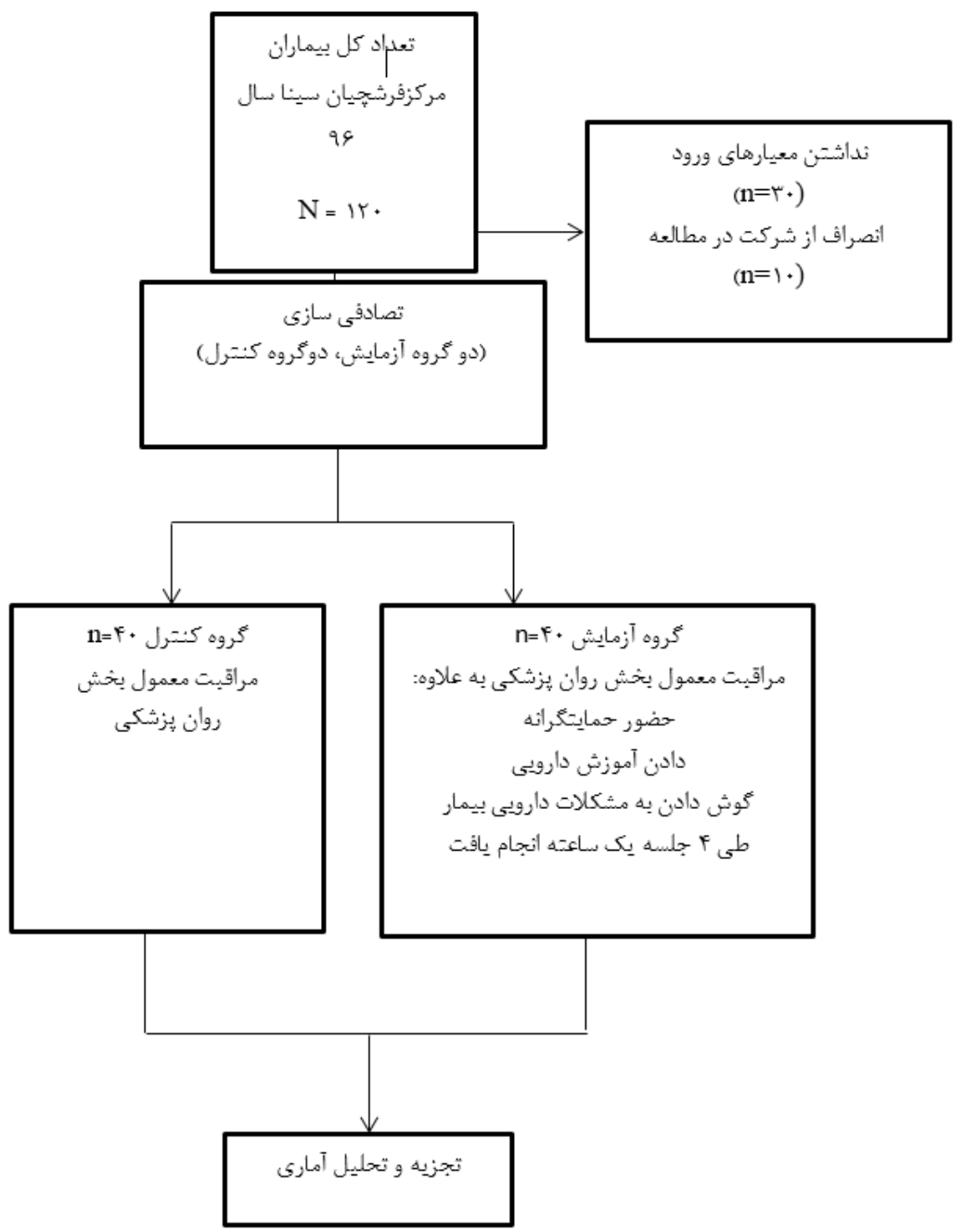

تصوير ا: تصوير كانسورت: روند انتخاب بيماران در مطالعه حاضر

همكن بودند. ميانگين و انحراف معيار تبعيت از رزيمم دارويى بيماران

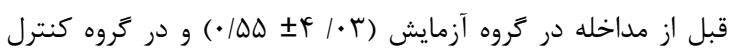

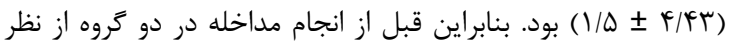

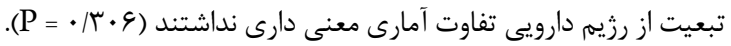

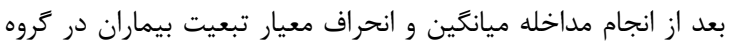

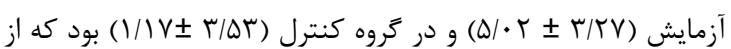

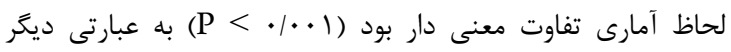

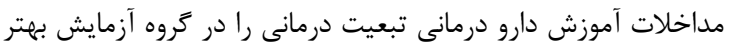

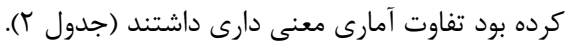

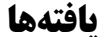

در طول مدت يروهش • r ا نفر وارد مطالعه شدند كه •r نفر شرايط

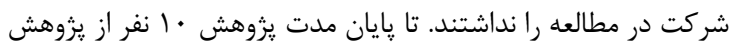

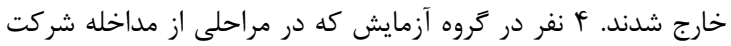

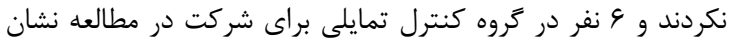

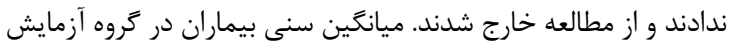
ك

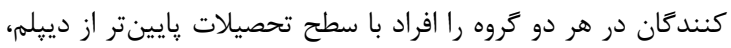

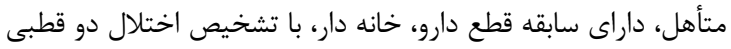

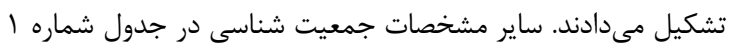

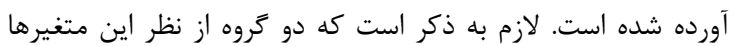




\begin{tabular}{|c|c|c|c|c|c|}
\hline & \multirow{3}{*}{$\begin{array}{c}\text { df } \\
1\end{array}$} & \multirow{2}{*}{$\mathbf{X}^{2}$} & \multirow{2}{*}{ كروه آزمايش تعداد (.) } & \multicolumn{2}{|c|}{ حدول r: مشخصات فردى بيماران با اختلالات روانى در گروه آزمايش و كنترل } \\
\hline \multirow{2}{*}{$\begin{array}{c}\text { P-value } \\
\cdot / \cdots\end{array}$} & & & & كروه كنترل تعداد (\%) & متغير \\
\hline & & $1 / \cdots$ & & & جنسيت \\
\hline & & & $(\Delta \cdot) r \cdot$ & $(\Delta \cdot) r \cdot$ & 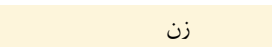 \\
\hline & & & $(\Delta \cdot) r \cdot$ & $(\Delta \cdot) r \cdot$ & مرد \\
\hline \multirow[t]{5}{*}{$\cdot|9 r|$} & r & $\cdot / 49$ & & & وضعيت تأهل \\
\hline & & & $(r \cdot)) \mathrm{r}$ & $(r \Delta)) \cdot$ & مجرد \\
\hline & & & $(f \cdot) \backslash \varepsilon$ & $(\uparrow \vee / D) \backslash \Lambda$ & 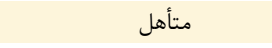 \\
\hline & & & $(T \Delta) l$. & $(T r / D) q$ & مطلقه \\
\hline & & & $(\Delta) r$ & $(\Delta) r$ & 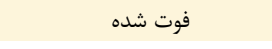 \\
\hline \multirow[t]{4}{*}{$\cdot|91|$} & r & $1 / V F$ & & & تحصيلات \\
\hline & & & $(\Delta \cdot) r \cdot$ & $(\Delta V / \Delta) r T$ & 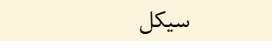 \\
\hline & & & $(\Gamma V / \Delta) \backslash \Delta$ & $(Y V / D) 11$ & 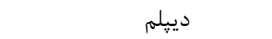 \\
\hline & & & $(\mid Y / \Delta) \Delta$ & $(\mid \Delta) \varphi$ & تحصيلات دانشگاهى \\
\hline \multirow[t]{5}{*}{. IATV } & r & $1 / V F$ & & & وضعيت اشتغال \\
\hline & & & $(F T / D) \backslash V$ & $(F Y / D) \backslash V$ & 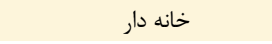 \\
\hline & & & $(1 \Delta) \varphi$ & $(V / \Delta) r$ & كارمند \\
\hline & & & $(I V / Q) V$ & $(1 \Delta) \varphi$ & بازنشسته \\
\hline & & & $(T \Delta))$. & $(r \Delta) \mid F$ & آزاد \\
\hline \multirow[t]{5}{*}{$1 / \cdots$} & r & $\cdot \cdots$ & & & تشخيص \\
\hline & & & $(T \Delta) 1$. & $(r \Delta)) \cdot$ & اسكيزوفرنى \\
\hline & & & $(r \cdot) \mid r$ & $(r \cdot) \mid r$ & دوقطبى \\
\hline & & & $(T \Delta) 1$. & $(T \Delta)) \cdot$ & افسردگى \\
\hline & & & $(T \cdot) \Lambda$ & $(T \cdot) \Lambda$ & اسكيزوافكتيو \\
\hline \multirow[t]{3}{*}{$\cdot 1199$} & 1 & $1 / 94$ & & & سابقه قطع دارو \\
\hline & & & $(q \vee / \Delta) r q$ & (9.) & بله \\
\hline & & & $(\Gamma / D) 1$ & $(1 \cdot)^{4}$ & خير \\
\hline \multirow[t]{5}{*}{$\cdot 190$} & r & $1 / 94$ & & & يِيحيرى درمان \\
\hline & & & $(\Delta \cdot) r \cdot$ & $(\Delta \cdot) r \cdot$ & 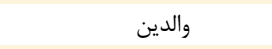 \\
\hline & & & $(r T / \Delta) \mid r$ & $(T \Delta))$. & همسر \\
\hline & & & $(I V / \Delta) V$ & $(Y T / Q)^{9}$ & ساير موارد \\
\hline & & & & & سن \\
\hline P-value & df & $\mathbf{T}$ & انحراف معيار & ميانگين & تروه \\
\hline \multirow[t]{2}{*}{$\cdot / V \cdot 1$} & vı & $\cdot / \pi \Lambda$ & $9 / 19$ & $r q / \Delta V$ & آزمايش \\
\hline & & & $9 / \% F$ & $r \Delta / v V$ & كنترل \\
\hline
\end{tabular}

جدول r: مقايسه ميانكين نمره تبعيت دارويى در گروه آزمايش و كنترل قبل و بعد از مداخله

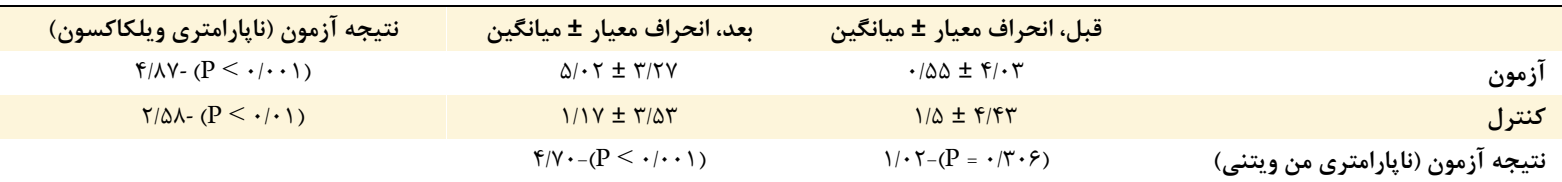

كافى از جانب كادر درمان ذكر كرده است[بها]. در مطالعه رحمانى و

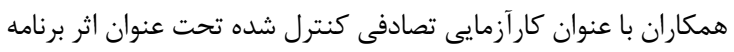

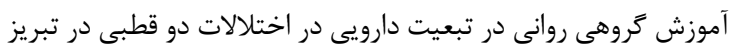

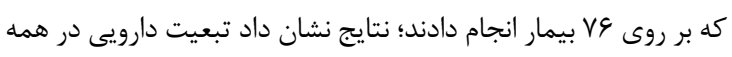

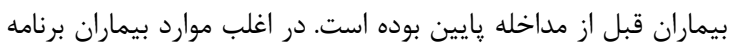

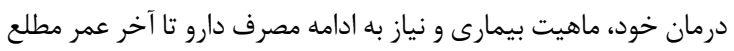

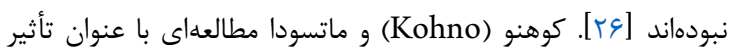

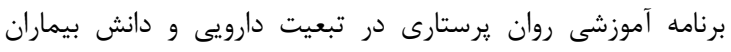

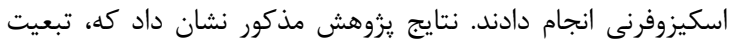

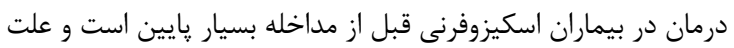

اين مطالعه با هدف بررسى تأثير آموزش دارويى بر تبعيت دارويى إنيى

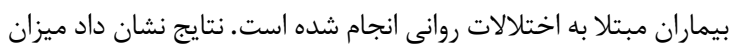

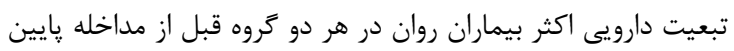

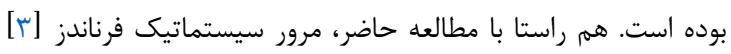

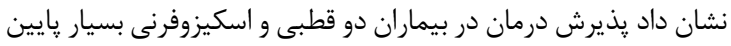

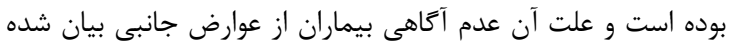

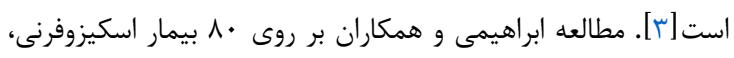

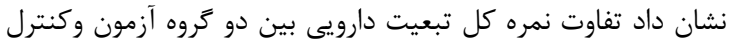

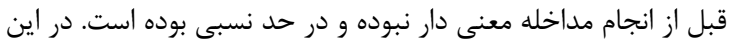

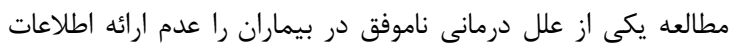


انتظارات آنها از كادر درمان است. بيماران بايد به راحتى انتقادات و وردات

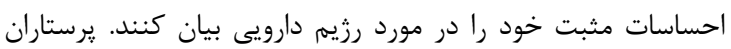

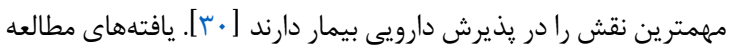

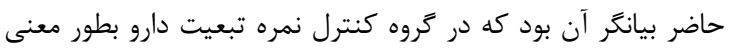

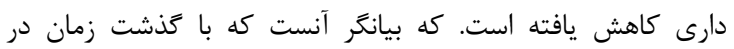

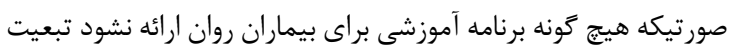

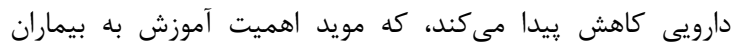

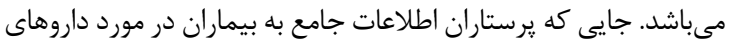

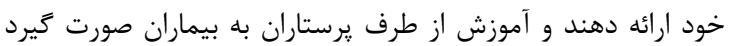

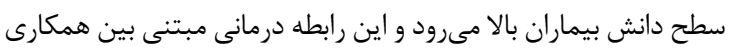

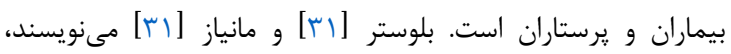

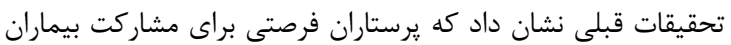

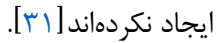

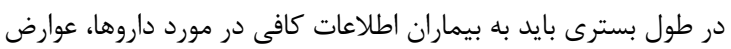

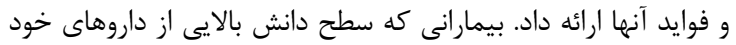

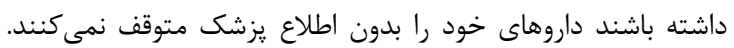

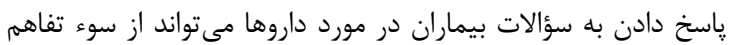

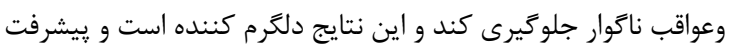

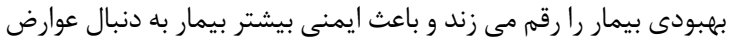

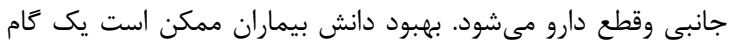

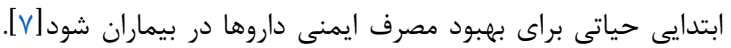

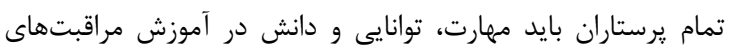

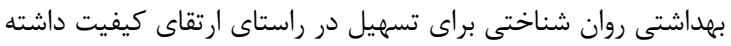

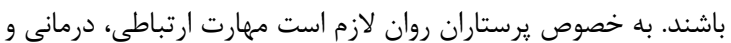

آموزشى بالايى داشته باشند [ [1].

\section{نتيجه تيرى}

طبق يافتهها مطالعه حاضر، آموزش دارو درمانى تبعيت دارويى بيماران

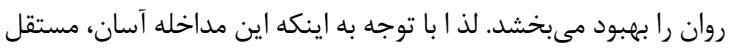

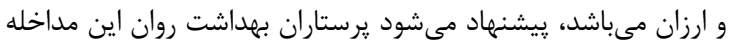

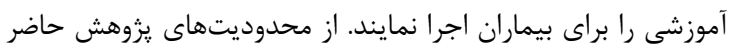

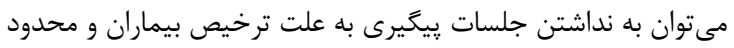

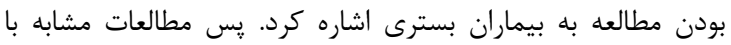

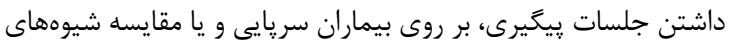

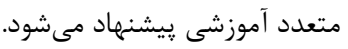

\section{سياسگزارى}

اين مقاله حاصل طرح تحقيقاتى مصوب پايان نامه كارشناسى ارشد

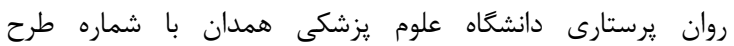
99.VTDFVIF IRCT2017071033374N4

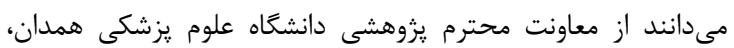

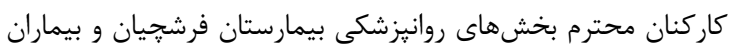

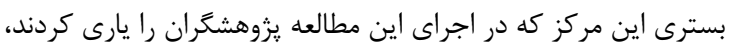
تشكر و قدردانى نمايند.

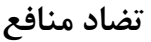

در اين مقاله تضاد منافع وجود ندارد.
آن عدم آكاهى بيماران از عوارض جانبى بيان شده است [ [ []. همجنين

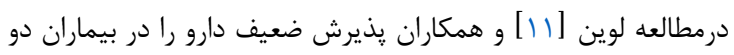

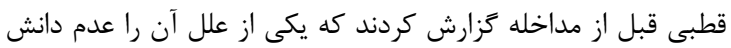

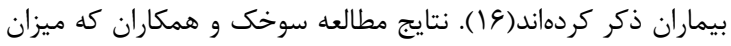

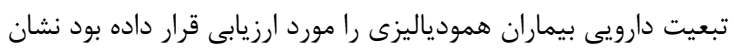

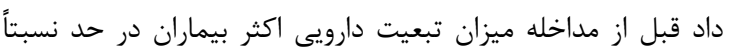

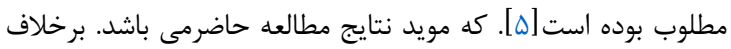

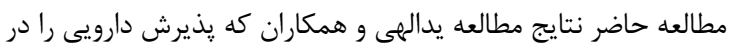

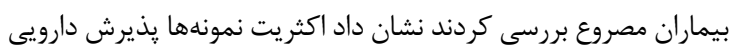

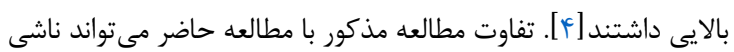

از تفاوت در جمعيت مورد مطالعه و ابزار مورد استفاده باشد.

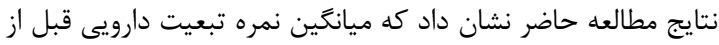

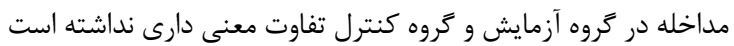

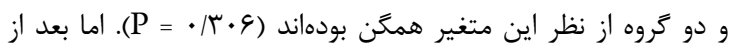

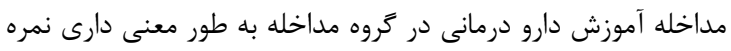
تبعيت دارويى افزايش يافته است (

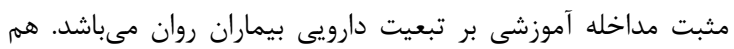

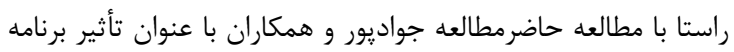

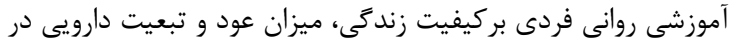

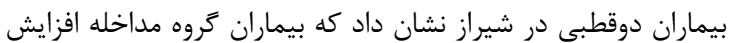

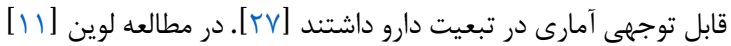

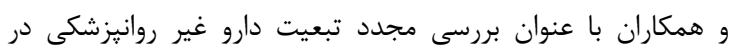

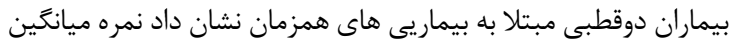

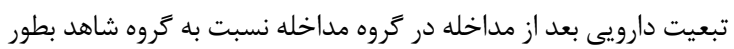
معنى دارى افزايش يافته است [ [1 1)].

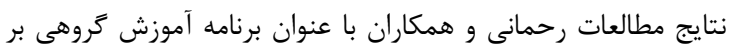

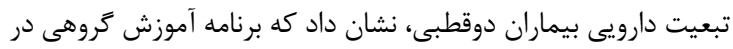

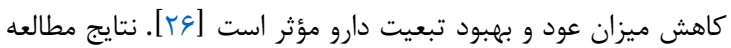

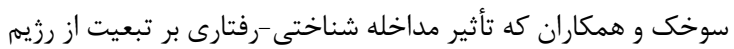

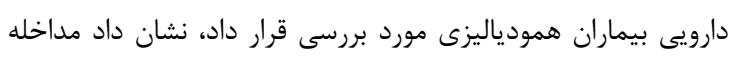

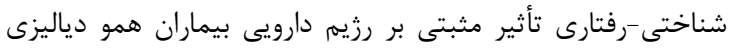

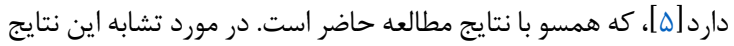

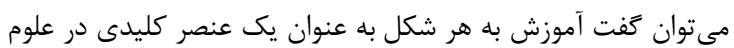

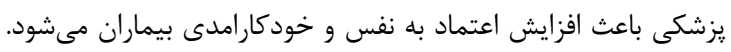

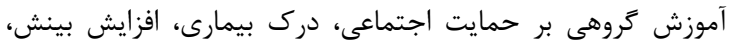

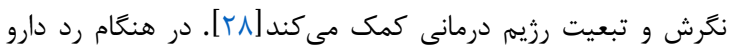

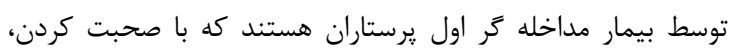

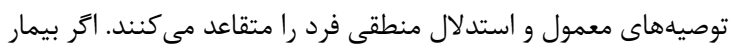

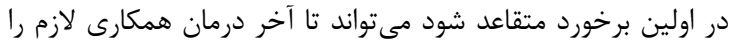

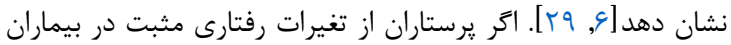

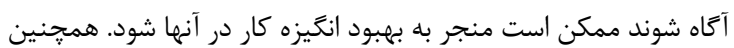

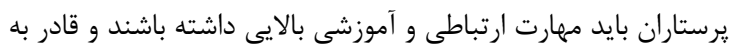

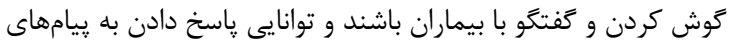

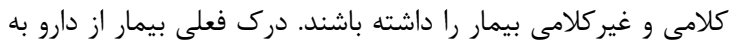

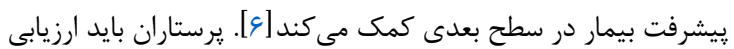

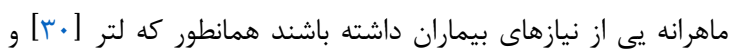

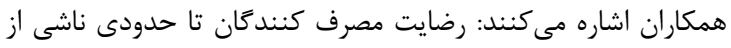




\section{References}

1. Ghorayshizadeh MA, Kouchaksaraei F, Pezeshki M. Evaluation of Risk Factors of Relapse in Bipolar I Disorders and Its Relation to Demographic Feature. Med J Tabriz Univ Med Sci. 2009;31(2):77-81.

2. Linsky A, Simon SR, Bokhour B. Patient perceptions of proactive medication discontinuation. Patient Educ Couns. 2015;98(2):220-5. doi:

10.1016/j.pec.2014.11.010 pmid: 25435516

3. Garcia S, Martinez-Cengotitabengoa M, Lopez-Zurbano S, Zorrilla I, Lopez P, Vieta E, et al. Adherence to Antipsychotic Medication in Bipolar Disorder and Schizophrenic Patients: A Systematic Review. J Clin Psychopharmacol. 2016;36(4):35571. doi: 10.1097/JCP.0000000000000523 pmid: 27307187

4. Yadollahi S, Ashktorab T, Zayeri F. Medication Adherence and Related Factors in Patients with Epilepsy. J Hayat. 2015;21(2):67-80. 5. Sookhak F, Zolfaghari M, Asadi Noghabi AA, Haghani H. Effect of a cognitive-behavioral intervention on medication adherence in patients' undergoing hemodialysis. J Hayat. 2014;20(3):8594.

6. Richardson M, Brennan G, James K, Lavelle M, Renwick L, Stewart D, et al. Describing the precursors to and management of medication nonadherence on acute psychiatric wards. Gen Hosp Psychiatry. 2015;37(6):606-12. doi: 10.1016/j.genhosppsych.2015.06.017 pmid: 26195348

7. Louis-Simonet M, Kossovsky MP, Sarasin FP, Chopard P, Gabriel V, Perneger TV, et al. Effects of a structured patient-centered discharge interview on patients' knowledge about their medications. Am J Med. 2004;117(8):563-8. doi: 10.1016/j.amjmed.2004.03.036 pmid: 15465504

8. Coe AB, Moczygemba LR, Gatewood SB, Osborn RD, Matzke GR, Goode JV. Medication adherence challenges among patients experiencing homelessness in a behavioral health clinic. Res Social Adm Pharm. 2015;11(3):e110-20. doi: 10.1016/j.sapharm.2012.11.004 pmid: 23218849 9. Dibonaventura M, Gabriel S, Dupclay L, Gupta S, Kim E. A patient perspective of the impact of medication side effects on adherence: results of a cross-sectional nationwide survey of patients with schizophrenia. BMC Psychiatry. 2012;12(1):20. doi: 10.1186/1471-244X-12-20 pmid: 22433036
10. Matsuda M, Kono A. Development and evaluation of a psychoeducation practitioner training program (PPTP). Arch Psychiatr Nurs. 2015;29(4):217-22. doi: 10.1016/j.apnu.2015.03.002 pmid: 26165976

11. Ebrahimi H, Namdar H. The effect of therapeutic relationship in schizophrenic patients. J Urmia Nurs Midwifery Fac. 2014;12(6):491-8.

12. Levin JB, Krivenko A, Bukach A, Tatsuoka C, Cassidy KA, Sajatovic M. A Reexamination of Nonpsychiatric Medication Adherence in Individuals With Bipolar Disorder and Medical Comorbidities. J Nerv Ment Dis. 2017;205(3):182-7. doi: 10.1097/NMD.0000000000000544 pmid: 27294318

13. Belzeaux R, Boyer L, Mazzola-Pomietto P, Michel P, Correard N, Aubin V, et al. Adherence to medication is associated with non-planning impulsivity in euthymic bipolar disorder patients. J Affect Disord. 2015;184(1):60-6. doi: 10.1016/j.jad.2015.05.041 pmid: 26070047

14. Shahin SH, Daly EB. Knowledge, attitudes and beliefs about psychotropic medication among Saudi hospitalized psychiatric patients. Int J Nurs Stud. 1999;36(1):51-5. doi: 10.2147/NDT.S56107 pmid: 10375066

15. El-Mallakh P, Findlay J. Strategies to improve medication adherence in patients with schizophrenia: the role of support services. Neuropsychiatr Dis Treat. 2015;11:1077-90. doi: 10.2147/NDT.S56107 pmid: 25931823

16. Costa E, Giardini A, Savin M, Menditto E, Lehane E, Laosa $\mathrm{O}$, et al. Interventional tools to improve medication adherence: review of literature. Patient Prefer Adherence. 2015;9(9):1303-14. doi: 10.2147/PPA.S87551 pmid: 26396502

17. Holman H. Chronic disease--the need for a new clinical education. JAMA. 2004;292(9):1057-9. doi: 10.1001/jama.292.9.1057 pmid: 15339897

18. Van Wijk BL, Klungel OH, Heerdink ER, de Boer A. Effectiveness of interventions by community pharmacists to improve patient adherence to chronic medication: a systematic review. Ann Pharmacother. 2005;39(2):319-28. doi: $10.1345 /$ aph.1E027 pmid: 15632223

19. Stevenson FA, Cox K, Britten N, Dundar Y. A systematic review of the research on communication between patients and health care 
professionals about medicines: the consequences for concordance. Health Expect. 2004;7(3):23545. doi: 10.1111/j.1369-7625.2004.00281.x pmid: 15327462

20. Gleeson T, Iversen MD, Avorn J, Brookhart AM, Katz JN, Losina E, et al. Interventions to improve adherence and persistence with osteoporosis medications: a systematic literature review. Osteoporos Int. 2009;20(12):2127-34. doi: 10.1007/s00198-0090976-0 pmid: 19499273

21. Schedlbauer A, Davies P, Fahey T. Interventions to improve adherence to lipid lowering medication. Cochrane Database Syst Rev. 2010(3):CD004371. doi: 10.1002/14651858.CD004371.pub3 pmid: 20238331

22. Schroeder K, Fahey T, Ebrahim S. Interventions for improving adherence to treatment in patients with high blood pressure in ambulatory settings. Cochrane Database Syst Rev. 2004;64(2):CD004804.

doi: 10.1002/14651858.CD004804 pmid: 15106262 23. Vergouwen AC, Bakker A, Katon WJ, Verheij TJ, Koerselman F. Improving adherence to antidepressants: a systematic review of interventions. J Clin Psychiatry. 2003;64(12):1415-20. pmid: 14728101

24. Firooz M, Mazlom SR, Hoseini SJ, Hasanzadeh F, KIMIAEE SA. Comparison between the effect of group education and group counseling on emotional self-care in type II diabetics. J Birjand Univ Med Sci. 2016;22(4):293303.

25. Sadeghian E, Afshar Mogadam F. Determination of factors affecting the medication compliance in patients admitted to psychiatric wards. J Qazvin Univ Med Sci. 2005;129(3):26-33. 26. Rahmani F, Ebrahimi H, Ranjbar F, Razavi SS, Asghari E. The Effect of Group Psychoeducation Program on Medication Adherence in Patients with Bipolar Mood Disorders: a Randomized Controlled Trial. J Caring Sci. 2016;5(4):287-97. doi: 10.15171/jcs.2016.030 pmid: 28032073

27. Javadpour A, Hedayati A, Dehbozorgi GR, Azizi A. The impact of a simple individual psycho-education program on quality of life, rate of relapse and medication adherence in bipolar disorder patients. Asian J Psychiatr. 2013;6(3):208-13. doi: 10.1016/j.ajp.2012.12.005 pmid: 23642977

28. Kunst EL, Mitchell M, Johnston AN. Manikin simulation in mental health nursing education: An integrative review. Clin Simulat Nurs. 2016;12(11):484-95.

29. Dankert ME, Brensinger CM, Metzger KL, Li C, Koleva SG, Mesen A, et al. Attitudes of patients and family members towards implantable psychiatric medication. Schizophr Res. 2008;105(1-3):279-86. doi: 10.1016/j.schres.2008.05.008 pmid: 18571376

30. Latter S, Yerrell P, Rycroft-Malone J, Shaw D. Nursing, medication education and the new policy agenda: the evidence base. Int J Nurs Stud. 2000;37(6):469-79. pmid: 10871657

31. Bolster D, Manias E. Person-centred interactions between nurses and patients during medication activities in an acute hospital setting: qualitative observation and interview study. Int J Nurs Stud. 2010;47(2):154-65. doi: 10.1016/j.ijnurstu.2009.05.021 pmid: 19577752 